\title{
FINITE ELEMENT ANALYSIS OF THE FLOW PATH OF FOOD 3D PRINTING NOZZLE
}

\author{
Yan Hao LI ${ }^{1}$, Guo Yun ${ }^{2}$ \\ ${ }^{1}$ Engineering training center, Shanghai University of Engineering Science, Shanghai, China \\ ${ }^{2}$ Engineering training center, Shanghai University of Engineering Science, Shanghai, China
}

\begin{abstract}
Dynamics characteristics of food fluid flow in a FDM (fused deposition) $3 D$ printer capsule tube, the existing $3 D$ printing nozzle structure was improved, and the mathematical model of flow velocity was established by combining the relevant theories of fluid mechanics. A fluid dynamics simulation model of the piston extrusion process was established by using ANSYS FLUENT, Through the analysis of the internal flow field distribution of the nozzle, the flow velocity of the nozzle section and the distribution of the internal pressure of the pipeline, the research shows that the improved nozzle can effectively alleviate the pressure field inside the nozzle and the flow field in the capsule tube more smoothly. The flow rate is stable step by step. This method provides a theoretical basis for studying the difficult problems of improving the $3 D$ printing extrusion process of food, and has certain reference value.
\end{abstract}

Keywords: Food fluid, Piston, Fluid mechanics.

\section{INTRODUCTION}

In the 21 st century, $3 \mathrm{D}$ printing technology is widely used in various fields to transform traditional material manufacturing into additive manufacturing, which greatly reduces material waste. The principle of $3 \mathrm{D}$ printing technology is actually not complicated. It is a technology based on digital model files, which uses an adhesive material such as powder metal or plastic to construct an object by layer-by-layer printing. Among them, the field of fused deposition molding (FDM) is the most widely used. FDM3D printing is that the material is heated and melted in the nozzle, the nozzle moves along the cross-sectional profile of the part and the filling trajectory, while the molten material is extruded, the material is rapidly solidified, and the surrounding material is Material bonding to achieve three-dimensional molding. The printer has many advantages such as simple structure, convenient operation and fast forming speed.

In recent years, 3D printing technology has begun to go to the fields of medicine, bioengineering, food, etc. In theory, $3 \mathrm{D}$ printers can print any complex shape of food through the movement of nozzles, and the ink cartridges of $3 \mathrm{D}$ printers can be customized to match the amount of nutrients required by individuals. Resulting in a richer, healthier and more controlled diet. It is more conducive to the use of new ingredients to create non-traditional foods that are not comparable to traditional food manufacturing processes. Therefore, 3D printing of food has become a new direction of 3D printing research. Farzad Liravi a, a scholar at the University of Waterloo abroad, established a prediction equation for the flow in $3 \mathrm{D}$ printing of highly viscous materials and verified the feasibility of the prediction equation through experiments. Lin wang, a scholar at Jiangnan University, studied the food printing materials of a surimi gel and passed the analysis of its rheology to verify the printing conditions and printing accuracy. Their research on food mobility has made a huge contribution to the follow-up study of food 3D printing. On the whole, the research at home and abroad at the current stage mainly focuses on the research on the $3 \mathrm{D}$ printing performance of food, and the research on the influence of the mechanical structure optimization of 3D printer on the flow ability of food is less. This paper is based on the analysis of food flow in the flow channel and nozzle structure of capsules containing food 3D printers based on ANSYS FLUENT, providing theoretical support for further optimization of design food $3 \mathrm{D}$ printers.

\section{3D PRINT NOZZLE PRINCIPLE}

\subsection{D Printer Discharging Method and Principle}

FDM 3D printing mainly uses extrusion to extrude food materials. For foods, this special edible material, there are three main types of mechanical extrusion: pneumatic extrusion, pushing food materials by gas; piston extrusion, piston extrusion is more stable and precise control than pneumatic extrusion, food materials are subject to The force is also more uniform; the screw extrusion method uses a screw feeding mechanism to extrude food materials. The accuracy is lower than the two extrusion methods above and requires more space. Therefore, this paper mainly studies the piston extrusion method, the main structure of which consists of push rod, piston, cylinder and nozzle. Its principle is shown in the fig 1 : 


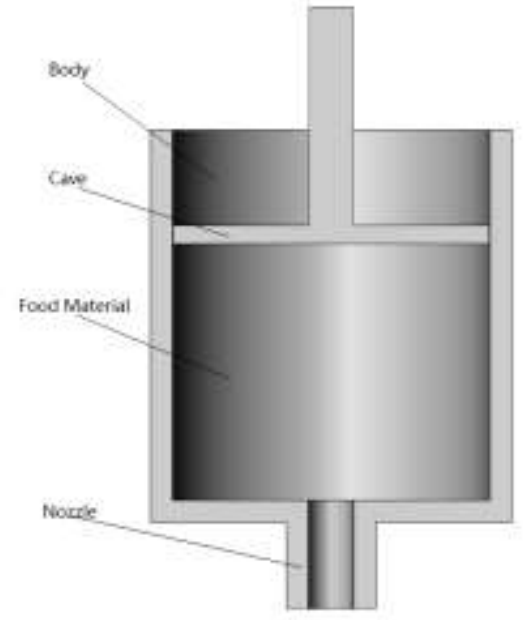

Fig 1: Piston nozzle schematic

\subsection{Cylinder Parameter Analysis}

The cylinder block is a key component in the FDM printer. Its main structure is a capsule tube and a spray head. Its working principle is to extrude the food material pushed by the piston through the capsule tube and the nozzle into the desired cross-sectional shape and size of the filament. This paper mainly studies the influence of the structure of the nozzle on the extrusion process of 3D printing.

\subsubsection{Flow Analysis of Food Materials in Cylinders}

The flow of food fluid in the cylinder clearly reflects the relationship between volumetric flow rate, pressure change and geometry. The food material is a non-Newtonian fluid, and the continuous equation and momentum equation for the fluid in the tube can be described as:

$$
\begin{gathered}
\frac{\partial \rho}{\partial \mathrm{t}}+\frac{\partial \rho u}{\partial x}+\frac{\partial \rho u}{\partial y}+\frac{\partial \rho u}{\partial z}=0 \\
\frac{\partial p \mathrm{u}}{\partial t}+\frac{\partial p \mathrm{u} \otimes u}{\partial x}+\frac{\partial p \mathrm{u} \otimes u}{\partial y}+\frac{\partial p \mathrm{u} \otimes u}{\partial z}=-\left(\frac{\partial p}{\partial x}+\frac{\partial p}{\partial y}+\frac{\partial p}{\partial z}\right)+\left(\frac{\partial \tau}{\partial x}+\frac{\partial \tau}{\partial y}+\frac{\partial \tau}{\partial z}\right)+p g
\end{gathered}
$$

$\tau$ —_ Stress tensor ;

$\rho=\rho(t) \_$Fluid density equation ;

g_ Gravity acceleration ;

$p=p(\rho, u) \_$Pressure equation ;

Food 3D printer capsules have slow food flow and small capsule size, so the flow can be regarded as steady flow and the compressibility is negligible.Food-based non-Newtonian fluids generally use a power law to express their rheological equations. Therefore, for the food material fluid in the cylinder, analyze its rheological equation:

$$
\tau=K\left(-\frac{d u}{d r}\right)^{n}
$$

(a pseudoplastic fluid when $\mathrm{n}<1$, and an expansive fluid when $\mathrm{n}>1$ )

$\mathrm{K}$ - Flow coefficient ;

$\frac{d u}{d r}$ Shear rate

In the calculation process in this equation, due to the small size of the capsule in this study, the size of the key parts is simplified, and the fluidity of the non-Newtonian incompressible fluid is analyzed.The fluid in the capsule tube meets:

$$
p \pi x^{2}=\tau 2 x \pi L
$$

$\mathrm{x}-$ Radius of the section ;

$\mathrm{L}-$ Length of runner ;

For the velocity distribution, simultaneous equations $2-3$ and 2-4 are available:

$$
\frac{p x}{2 L}=K\left(-\frac{d u}{d x}\right)^{n}
$$

Separate variable solution:

$$
d u=-\left(\frac{p x}{2 L K}\right)^{1 / \mathrm{n}} d x
$$

Solving the integral of its equation

$$
\begin{array}{r}
\int_{0}^{u} d u=-\int_{0}^{x}\left(\frac{p x}{2 L K}\right)^{1 / n} d x \\
u=\frac{R(R p)^{1 / n}-x(x p)^{1 / n}}{(1 / n+1)(2 K L)^{n}}+C
\end{array}
$$

Since the flowing liquid in the tube is a power law food fluid, the flow is a steady state flow, and the fluid has no relative slip to the capsule wall,And $u=\left.0\right|_{x=R}$ there is $\mathrm{C}=0$, so the flow rate $\mathrm{Q}$ in the capsule can be obtained:

$$
Q=\frac{\pi n R^{3}}{3 n+1}\left(\frac{P R}{2 L K}\right)^{1 / n}
$$

This is the relationship between the flow rate of the power law fluid layer and the pressure drop.

From the above formula, the average flow velocity of the section can be obtained as v:

$$
v=\frac{Q}{\pi R^{2}}=\frac{n R(R p)^{1 / n}}{(2 L)^{1 / n}(3 n+1)}
$$


The cross-section flow is calculated by the simulation equation, which provides a theoretical basis for the effective optimization of the design of the nozzle structure.

\subsubsection{Nozzle Flow Channel Structure Design}

For the structure design of the nozzle, this paper mainly studies the traditional extrusion nozzle and the optimized nozzle. The design of the conventional nozzle is mainly for the rapid extrusion structure as shown in Fig.1.This structure is the most common structure in the extrusion type, and the internal structure is simple and easy to process. Based on Figure 2 and the above flow calculations, the size is initially determined according to Table 1 .

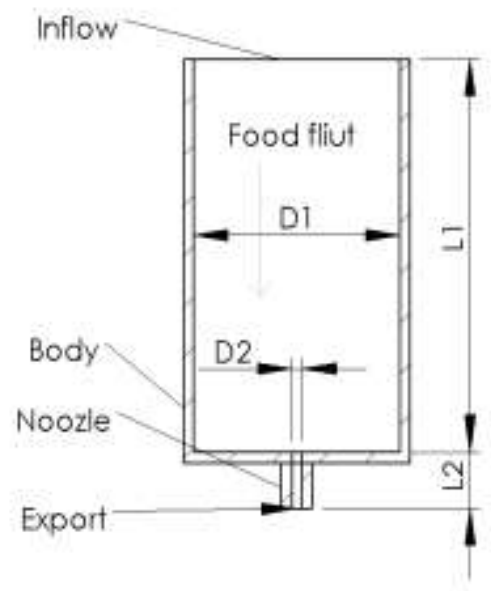

Fig 2: Common type nozzle structure diagram

Table 1: Common type flow channel design parameters

\begin{tabular}{|l|l|l|l|}
\hline D1 & D2 & L1 & L2 \\
\hline 40 & 5 & 150 & 45 \\
\hline
\end{tabular}

Transforming the traditional structure, the optimized nozzle flow channel structure is optimized for the retention interval that is easily generated at the right angle structure at the corner of the conventional structure. The food material is a non-Newtonian fluid, the fluid density is large, and the food material flows poorly at the corners, which is prone to particle deposition. In this paper, we retrofit these defects and use the pattern of excessive stratification and depressurization to study whether the structure of stratified depressurization can effectively improve the fluidity of food materials. Based on Fig.3 and the above flow calculations, preliminary determinations are made according to Table 2 .

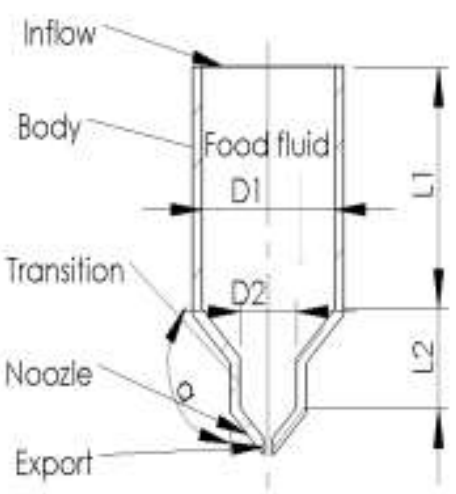

Fig 3: Improved nozzle structure

Table 2: Improved type flow channel design parameters $(\mathrm{mm})$

\begin{tabular}{|l|l|l|l|l|l|l|}
\hline D1 & D2 & D3 & L1 & L2 & L3 & a \\
\hline 40 & 15 & 5 & 150 & 30 & 15 & $120^{\circ}$ \\
\hline
\end{tabular}

\section{FINITE ELEMENT ANALYSIS OF FOOD EXTRUSION NOZZLE}

\subsection{Food Material Analysis and Motion Analysis}

For edible materials, most of the fluids are non-Newtonian fluids, wherein the food fluids are mainly three pseudoplastic fluids, expansive fluids, and Bingham fluids. This paper focuses on pseudoplastic food fluids in which the viscosity of chocolate decreases with increasing shear rate. The food fluid is pushed by the push rod to push out the food material inside the capsule, In order to ensure the fluidity of chocolate, which is sensitive to temperature, the heating of the capsule is controlled by full pipe thermostat. For the flow analysis of food fluids, the Reynolds number $\mathrm{Re}$ of the food fluid during the actual flow in the piston print nozzle is:

$$
\operatorname{Re}=\frac{v d}{V}
$$

$\mathrm{V}$ - Fluid kinematic viscosity ;

d-Channel feature length ;

$\mathrm{v}-$ Average cross section speed. ;

For the food-based 3D printer capsules studied in this paper, the diameter $\mathrm{d}$ is generally between 40 and $100 \mathrm{~mm}$, the fluid velocity is generally lower than $5 \mathrm{~mm} / \mathrm{s}$, and the viscosity of the food hydrodynamic viscosity $\mathrm{v}$ in the printable temperature range is generally greater than 1 $\mathrm{Pa} \cdot \mathrm{s}$..Therefore, the Reynolds number is much less than 2300 , and the fluid in this paper is treated as laminar flow. The capsule conduit device is cylindrical, so the extrusion process of the food $3 \mathrm{D}$ printing piston nozzle can be simplified to the liquid laminar flow in the round tube. 


\subsection{Finite Element Analysis of Different Nozzle}

\section{Structures}

For the common and improved physical models, the numerical simulation of the extrusion process was carried out by using the finite element simulation ANSYS FLUENT software. The material chosen is chocolate food fluid material. However, the densities of the chocolate fluids under different formulations are different. Therefore, the valuation method is adopted, and the density is about 1750 $\mathrm{kg} / \mathrm{m} 2$ based on the optimization model. The viscosity of the chocolate hydrodynamic viscosity in the use temperature is about $2.35 \mathrm{~Pa} \cdot \mathrm{s}$. The inlet speed is $2 \times 10^{\wedge}-3 \mathrm{~m} / \mathrm{s}$. The outlet pressure is 0 at the standard atmospheric pressure, 1 standard atmospheric pressure is used as the reference pressure, the wall thermodynamic temperature $0 \mathrm{~K}$ is the reference temperature, the wall-free sliding boundary condition is adopted, and the flow field mode is laminar.
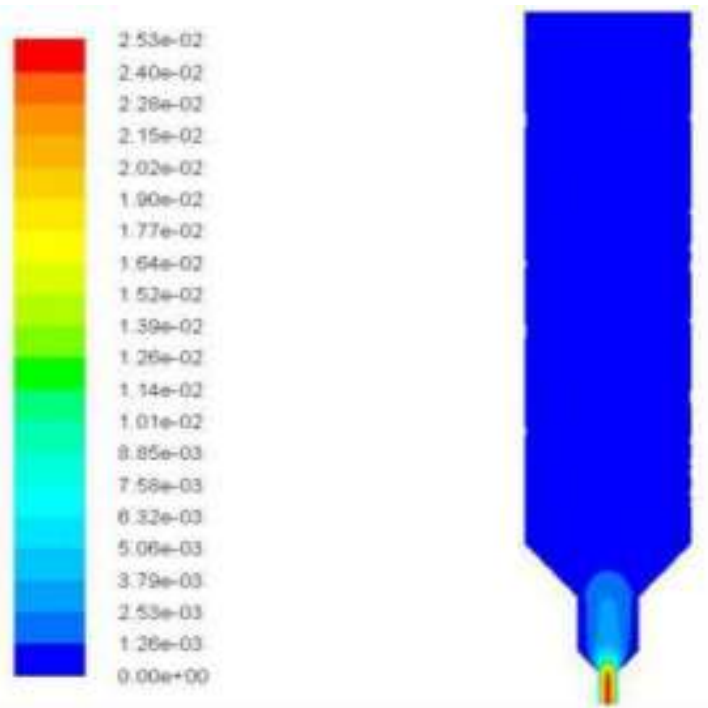

Fig 4: Improved speed cloud
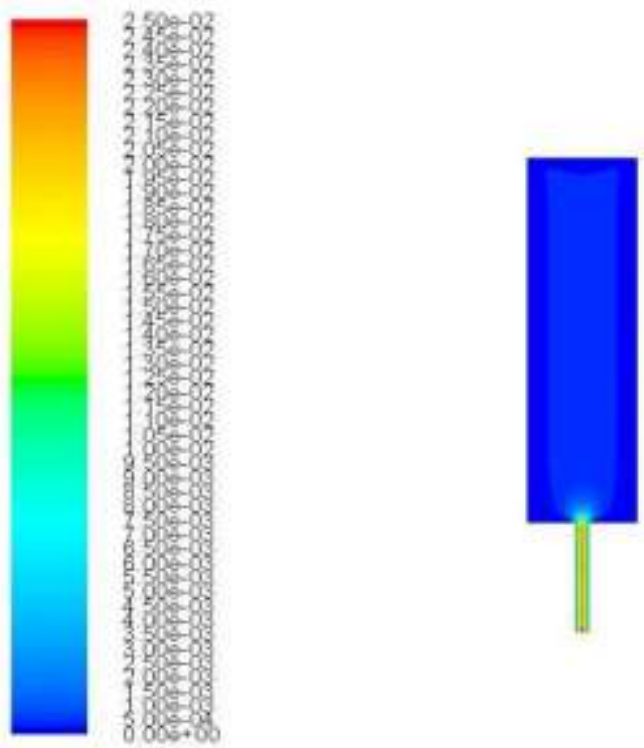

Fig 5: Common type speed cloud
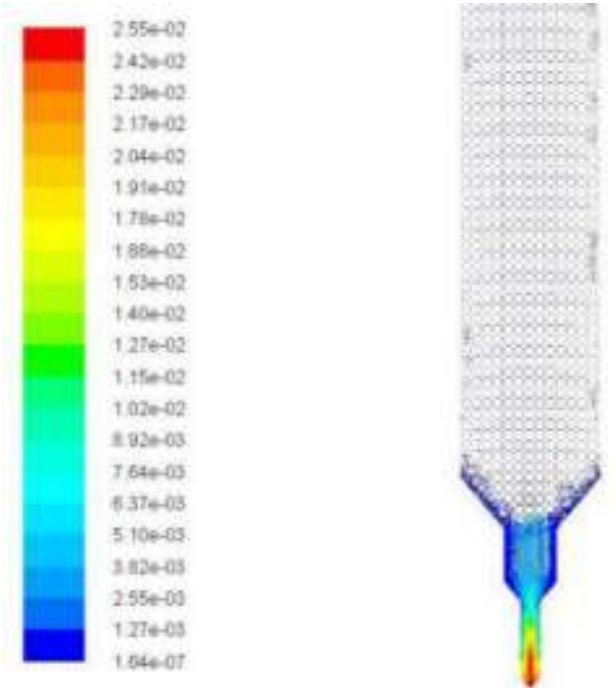

Fig 6: Improved speed vector cloud

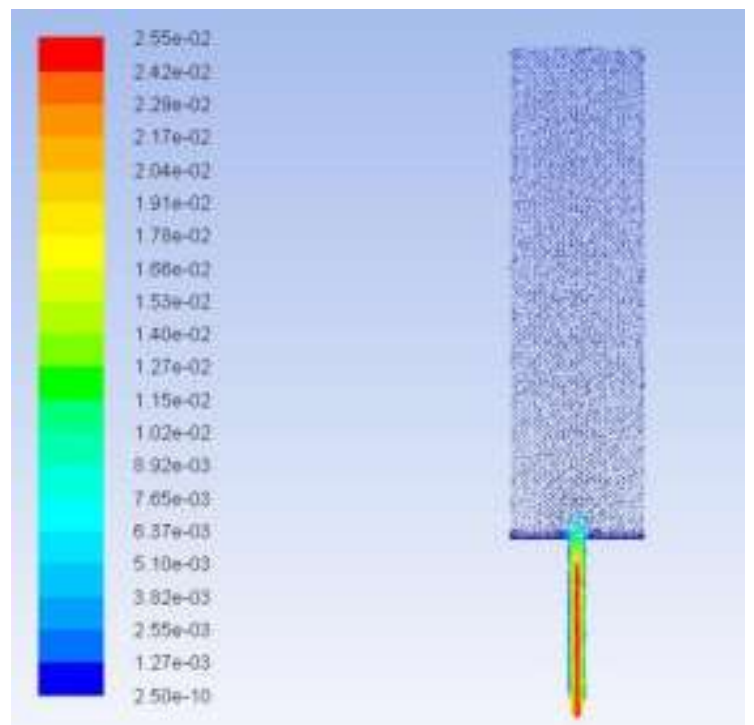

Fig 7: Common type Speed vector cloud

After the ANSYS FLUENT software simulation analysis, the speed cloud and velocity vector cloud maps of the flow velocity of the two different structural cylinders are analyzed by the simulation results. It can be seen that the common type structure exists at the right-angle corner wall of the upper and lower joints of the capsule and the nozzle compared with the improved structure. The apparent velocity retention, and the velocity vector direction are not concentrated at the nozzle, The velocity and vector cloud diagrams of the commonly used nozzles show that the motion of the fluid is mainly concentrated near the axis of the capsule tube, the fluidity is close to the wall, and the fluids in the two regions are significantly different. During the long-term extrusion process, this area will produce a backlog of food fluids, moisture will be lost in advance, and liquid phase separation will occur, causing printer failure and printing accuracy. The fluid flow rate is uniform in the improved nozzle overall mechanism. 


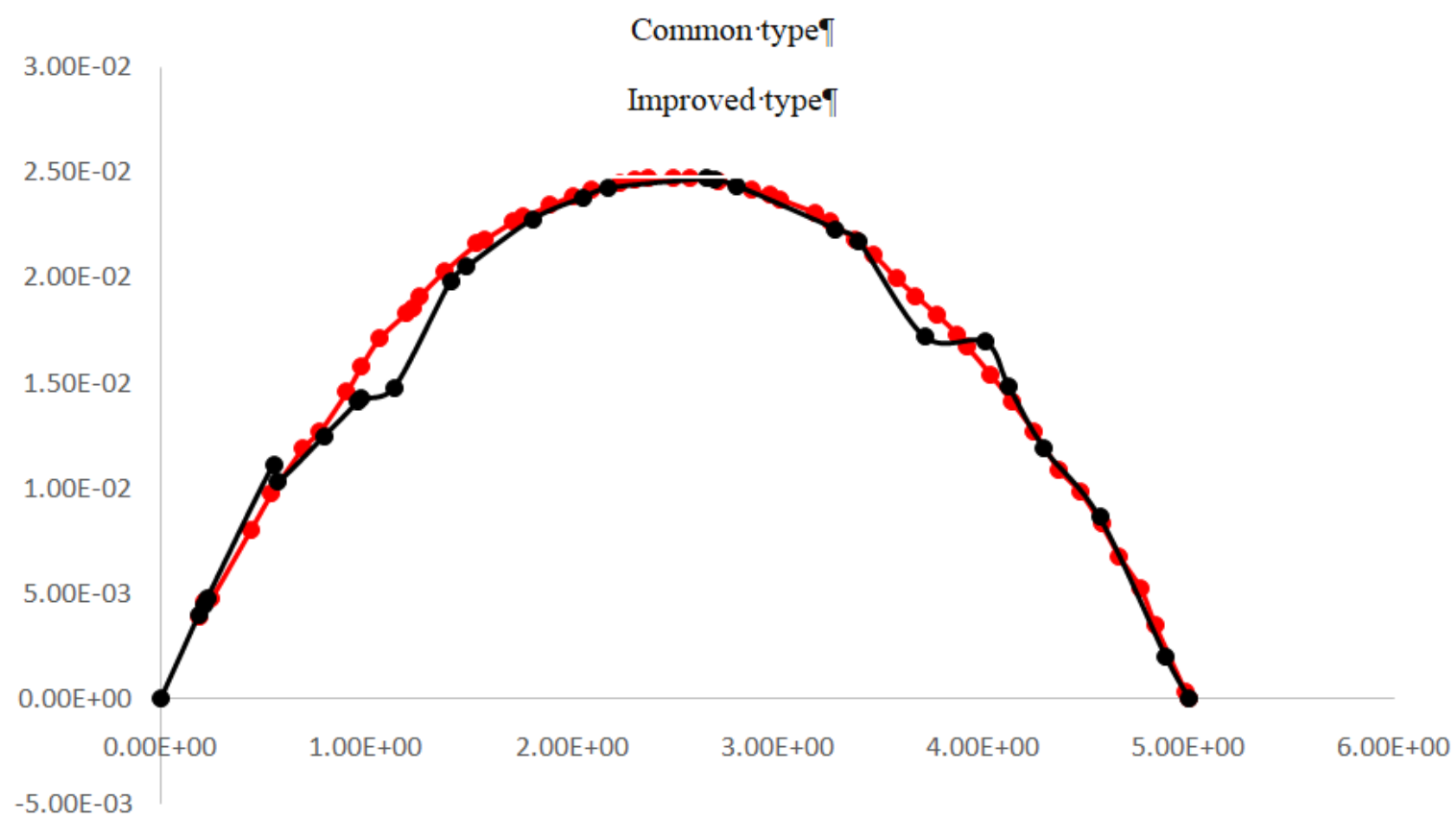

Table 3: Exit section flow rate diagram $\mathrm{X}$-axis is Nozzle section coordinates $\mathrm{Y}$-axis is velocity

Table. 3 mainly shows the flow rate of the food fluid in the joint port at any position of the outlet section in the two printings. The analysis chart shows that the highest flow velocity of the common type is $0.0250 \mathrm{~m} / \mathrm{s}$, and the modified fluid maximum velocity is $0.0252 \mathrm{~m} / \mathrm{s}$. The crosssectional flow velocity of the structure has a high similarity, but the flow velocity of the improved fluid at each position is more stable and the curve is stable. The fluid data points at various positions of the cross-section in the FLUENT simulation are more dense, indicating the cross-section in the finite element simulation. The speed of each point is stable, and the position speed of the common type structure node is selected less, and the oscillation is more unstable, which has a certain influence on the shape of the nozzle wire in $3 \mathrm{D}$ printing, which will cause the printing precision to be lowered.
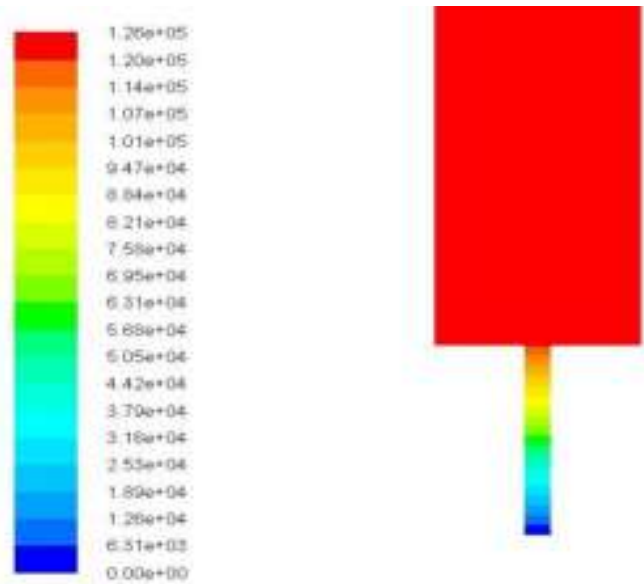

Fig 8: Common type pressure cloud map

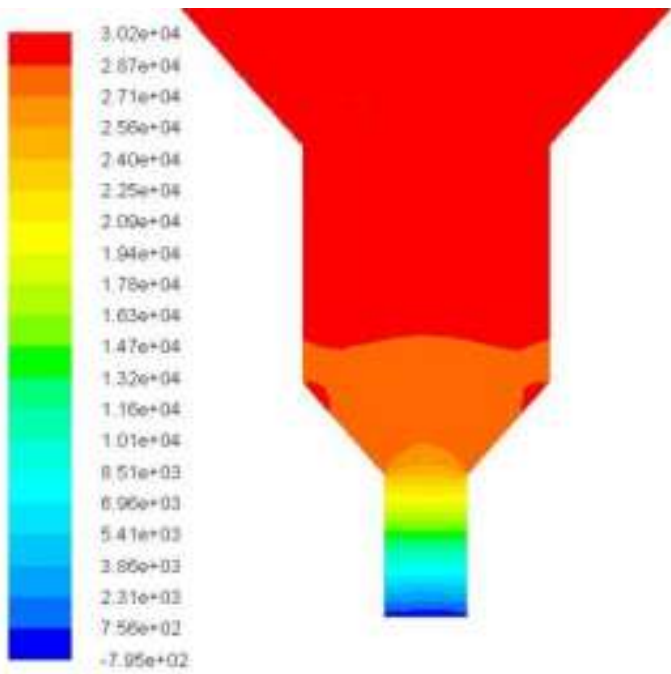

Fig 9: Improved pressure cloud map

From the pressure cloud diagram, the maximum pressure of the common type nozzle is $1.26 \times 105 \mathrm{~Pa}$, and the maximum pressure of the improved type is $3.02 \times 104 \mathrm{~Pa}$. The maximum pressure of the common type is much larger than that of the improved one. The reduction of the internal maximum pressure can effectively reduce the material of the nozzle. The damage, and the viscosity of the food fluid is mostly affected by the pressure. In this paper, the viscosity of the selected food fluid is simplified to account for the viscosity change caused by the pressure. The actual printing pressure will have a significant impact on the viscosity of the food. This directly affects the extrusion and printing accuracy of the print. 


\section{CONCLUSION}

During the Food fluid extrusion process, the design of the inside of the extrusion head has a certain influence on the printing, which will directly affect the smoothness of the extrusion process and the surface precision in the printing process. Therefore, the study of the printed structure in this paper is based on fluid mechanics. Guided by the rapid and free flow of food fluid in the flow channel, the numerical simulation analysis of the two structures shows that the common nozzle structure is simple and easy to process, but the flow field of the food fluid is unstable in the piston extrusion. The internal pressure field is higher, which increases the burden on the $3 \mathrm{D}$ printer, and the outlet crosssection flow rate fluctuates greatly, which causes the printing precision to decrease. The improved structure capsule body has a relatively stable flow rate and the minimum internal pressure, and the flow rate at the nozzle. Uniform change for more optimized 3D printing. It provides a basis for the optimal design of food fluid 3D printers with a certain viscosity.

\section{REFERENCES}

[1]. Jeffrey I. Liptonetc. "Additivemanufacturing forthe food industry, Trends in Food Science \& Technology," 43, 114-123, 2015

[2]. Steve Grahametc. "Classification of the printability of selected food for 3D printing:Development of an assessment method usinghydrocolloids asreference material,"Journal of Food Engineering ,215 ,23-32,2017.

[3]. Isaac Alves Ferreira etc. "Low-cost 3D food printing," Ciência \& Tecnologia dos Materiais ,29 , e265-e269.2017

[4]. Fan Yang eyc. "Recent development in 3D food printing," CRITICAL REVIEWS IN FOOD SCIENCE AND NUTRITION, VOL.57,3145-3153,2017

[5]. Chen Li De. "Strength, fracture and compression properties of gelatins by a new 3Dprinted tool," Journal of Food Engineering, 1-11,2017.

[6]. Frank Handle."Extrusion in ceramics[M],"Berlin : SpringerVerlag Berlin and Heidelberg GmbH \& Co,2009.

[7]. Wang Jiwen. "Solid freeform fabrication of biological materials [D] ,"Storrs:Graduate University of Connecticut, 2006.

[8]. hu Dongbin, Liang Jinping,Qu Yunxia.“ Functionalized bioartifact fabricated via selective slurry extrusion[J],"Journal of Nanoscience and Nanotechnology, 14,3703-3706,2014,

[9]. Zhang Daiquan, Chen Guoping. "The numerical simulation forextrusion forming of magnesium alloy pipes [J],'PhysicsProcedia, $25: 125-129,2012$.

[10]. Suhane, A. "Research on Micro-flow Extrusion Head of Denture Ceramic Slurry," JOURNAL OF MACHINE DESIGN, vol. 33,2, 2012. 